\title{
Software galore and high-resolution diffraction
}

POWDER DIFFRACTOMETER PANalytical

\author{
wWw.panalytical.com
}

X'pert PRO Alpha-1

PANalytical claim that the new X'pert PRO Alpha-1 $\mathrm{X}$-ray powder

diffractometer comes close to synchotron quality. It includes a symmetrical incident beam Johansson monochromator and the ultrafast X'Celerator detector. The company says that the monochromatic diffractograms obtained with this instrument have an excellent peak-tobackground ratio and a resolution that can only be improved by synchrotron diffractometers equipped with analyser crystals. A scan recorded overnight or during the weekend is sufficient for obtaining data with a quality that used to come from synchrotron

diffractometers only. The figure below shows results from an overnight scan on the NIST standard material SRM660a (lanthanum hexaboride); the insert shows the first reflection of $\mathrm{LaB}_{6}$.

An advantage of this instrument is that it can rapidly be reconfigured to another X-ray optical setup thanks to the PreFIX concept. This means, for example, that the system can simply and easily be changed from a parafocusing setup to a Debye-Scherrer geometry, in which the powder sample is in a tiny glass capillary. This is especially useful for organic samples, where needle-shaped or plate-like crystallites make a random orientation in a flat sample almost impossible.

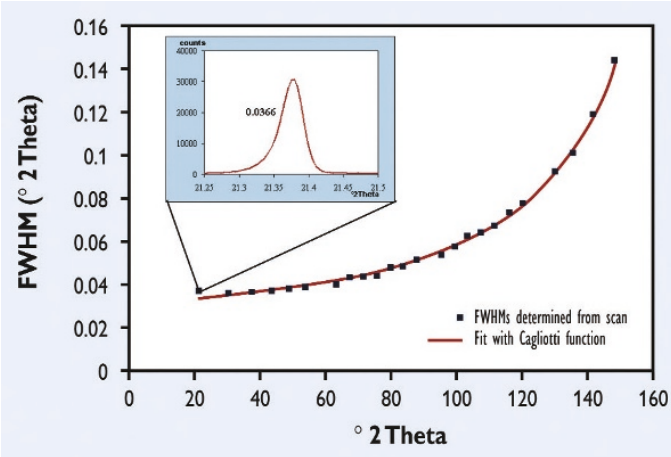

MICROANALYSIS SOFTWARE EDAX ww.edax.com

GENESIS 3.5

The new GENESIS 3.5 software from EDAX supports their new digital pulse processing (DPP II) hardware. In addition to existing GENESIS features, multiple spectrum overlays, a mapping view mode enabling up to 36 images to be displayed - and a stub view mode for easy view of multifield maps are offered. The company says that the DP II hardware offers up to $40 \%$ increased throughput and can handle over 150,000 counts per second.

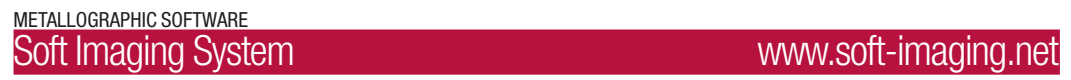

analySIS

Soft Imaging System announces a new series of software packages in the analySIS range, designed specifically for materials science, and to simplify standard workflows in metallographic

laboratories. They all offer intelligent image acquisition, wellstructured data storage, and automatic report generation. LabFLow also provides archiving and documentation, in compliance with common inspection and lab routines. The software controls motorized microscopes and/or stages. The analySIS CastIron Solver package analyses the shape and size of cast iron automatically or manually, and determines the ferrite/pearlite ratio in accordance with international and national standards; graphite can also be analysed. The Steel Factory package evaluates and documents grain size according to the intercept and planimetric methods; Steel Solver supports the intercept method only. The analySIS Materials Research Lab software combines materials analysis evaluation methods into one software solution that is compliant with national and/or international norms. Solutions for the following are included: automatic analysis of
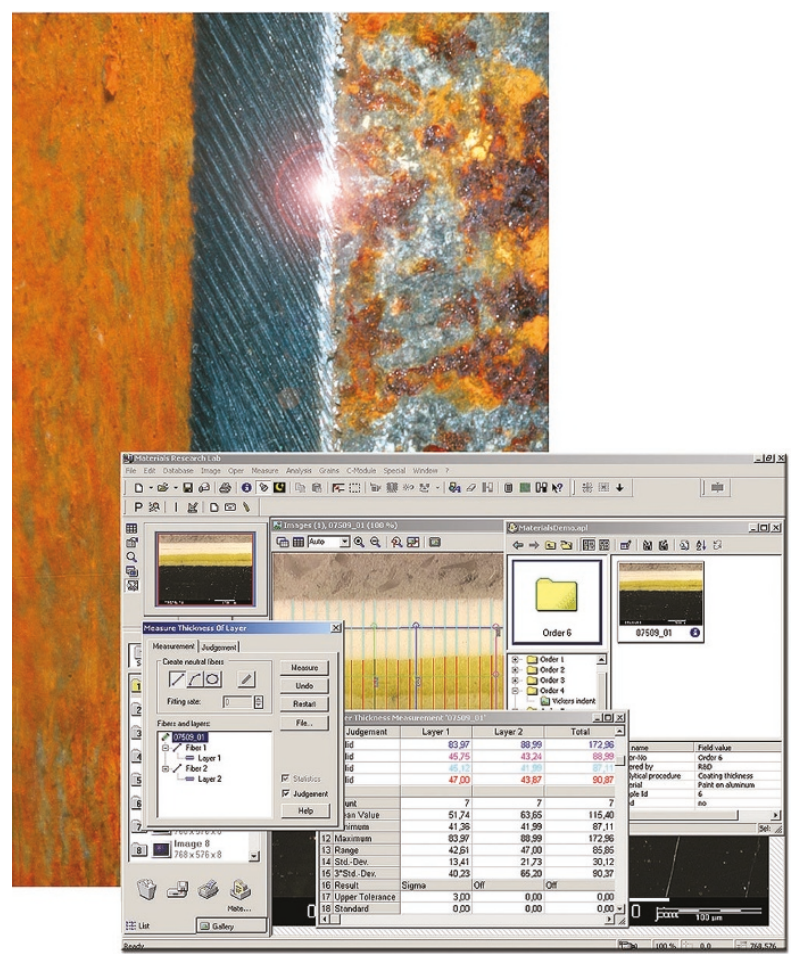

grain sizes; determining graphite content in cast iron; evaluating micro hardness indents; measuring layer thickness; determining surface roughness; digital chart comparison; automatic metallographic particle analysis and classification; class analysis; and phase analysis.

There is also a new add-in for the analySIS range: Itm (layer thickness measurement), a tool for determining layer thickness when analysing porous or compact coatings; it also offers crack width. Single and multiple coatings for a cut specimen can be measured using the concept of neutral fibres. The measurement is executed perpendicular to the user-definable neutral fibres (straight lines, curves or circles can be neutral fibres) meaning that complex coating structures and any modelled surfaces can be evaluated. The crack or shape of contours of arbitrarily shaped surfaces can be tracked and data results immediately provided. The thickness for each layer, average values, minimum and maximum values or standard deviation and a tolerance control are available. precise measurement of

These notes are compiled in the Nature Materials office from information provided by the manufacturers. 